\title{
Langzeiteffektivität von Baricitinib bei atopischer Dermatitis belegt
}

Eine Langzeittherapie mit Baricitinib kann die nach 16 Wochen erreichten Verbesserungen der atopischen Dermatitis bei Erwachsenen mit moderaten bis schweren Formen einer US-Studie zufolge über 68 Wochen aufrechterhalten.

\begin{abstract}
Der orale selektive Januskinase-Inhibitor Baricitinib hat seine Wirksamkeit über 16 Wochen bei Patienten mit moderater bis schwerer atopischer Dermatitis in Phase-III-Studien (BREEZE-AD1 und BREEZE-AD2) bereits belegt. Nun haben Prof. Dr. Jonathan Silverberg von der George Washington University School of Medicine und Kollegen die Langzeiteffekte bei Erwachsenen untersucht [1]. Dazu wurden diejenigen, die in den beiden Vorläuferstudien vollständig (Responder, vIGA-AD-Score 0 oder 1, keine Notfalltherapie) oder teilweise (TeilResponder, vIGA-AD 2, keine Notfalltherapie) auf die Therapie angesprochen hatten, für weitere 52 Wochen in die multizentrische, doppelblinde BREEZE-AD3-Studie eingeschlossen. Die Studienteilnehmer erhielten wie in den vorherigen Studien Baricitinib 2 mg (54 Personen) oder 4 mg (70 Personen).
\end{abstract}

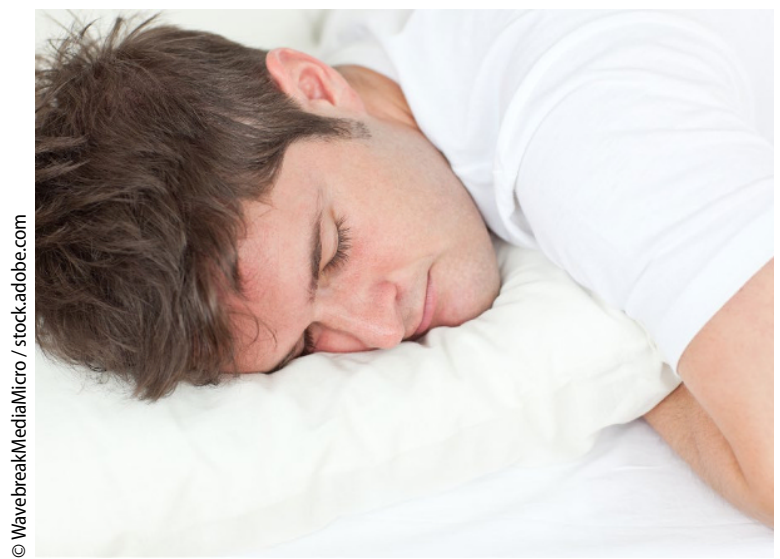

Abb. $1 \Delta$ Die Verbesserung des Schlafs blieb mit beiden Dosierungen und zu beiden Zeitpunkten unter der jeweiligen Therapie relativ konstant

\section{BREEZE-AD3-Studie}

In der 4-mg-Gruppe war der vIGA-AD (validated Investigator Global Assessmant for Atopic Dermatitis) unter kontinuierlicher Therapie während der gesamten Studienzeit stabil; einen Wert von 0 oder 1 hatten $45,7 \%$ in Woche 16 und 47,1\% in Woche 68 . Eine Verbesserung von $\geq 75 \%$ im EASI(Eczema Area and Severity Index, EASI75)-Score erreichten in Woche 16 insgesamt 70,0\% der Teilnehmer, in Woche 68 war ihr Anteil leicht auf $55,7 \%$ gefallen. Eine Verringerung des Juckreizes (NRS, $\geq 4$ Punkte vom Ausgangswert) wurde in Woche 16 bei $52,5 \%$ und in Woche 32 bei $45,9 \%$ registriert.

\section{》) Auch die Schmerzreduktion}

blieb mit beiden Dosierungen relativ konstant

In der 2 mg-Gruppe erreichten $46,3 \%$ in Woche 16 und 59,3\% in Woche 68 den vIGA-AD von 0 oder 1. Der Anteil der Patienten mit EASI75 stieg von $74,1 \%$ in Woche 16 auf $81,5 \%$ in Woche 68 , und der Juckreiz war in Woche 16 bei $44,2 \%$ und in Woche 32 bei $39,5 \%$ der Patienten entsprechend den Vorgaben verringert.

\section{Schmerzreduktion und Verbesserung des Schlafs}

Auch die Schmerzreduktion und die Verbesserung des Schlafs blieben mit beiden Dosierungen und zu beiden Zeitpunkten unter der jeweiligen Therapie relativ konstant (Skin pain NRS Verbesserung $\geq 4$ Punkte: $4 \mathrm{mg}$ : 61,8 \% bzw. 54,5 \%; 2 mg: 47,5 \% bzw.
45,0 \%; Atopic Dermatitis Sleep Scale, ADSS, Verbesserung $\geq 1,5$ Punkte: $4 \mathrm{mg}: 75,0 \%$ bzw. $71,4 \%$; $2 \mathrm{mg}$ : $73,7 \%$ bzw. 73,7 \%) (• Abb. 1). Den leichten Rückgang des Patientenanteils mit Besserung von Juckreiz und Schmerzstärke schreiben Silverberg und Kollegen einer veränderten Symptomwahrnehmung im Zeitverlauf zu.

Diese Ergebnisse, so Silverberg und Kollegen, belegten die Langzeitwirksamkeit von Baricitinib in den Dosierungen $4 \mathrm{mg}$ und $2 \mathrm{mg}$ für Patienten mit moderater bis schwerer atopischer Dermatitis.

\section{Literatur \\ 1. Silverberg Jl et al (2021) Long-term Efficacy of Baricitinib in Adults With Moderate to Severe Atopic Dermatitis Who Were Treatment Responders or Partial Responders: An Extension Study of 2 Ran- domized Clinical Trials. JAMA Dermatol 157(6):691-699. https://doi.org/10.1001/ jamadermatol.2021.1273.}

Hinweis des Verlags. Der Verlag bleibt in Hinblick auf geografische Zuordnungen und Gebietsbezeichnungen in veröffentlichten Karten und Institutsadressen neutral.

hautnah $2021 \cdot 20: 128$

https://doi.org/10.1007/s12326-02100459-1

(c) Springer-Verlag GmbH Austria, ein Teil von Springer Nature 2021 